\title{
Coping with COPE
}

\author{
Adeleke Victor Adedayo ${ }^{1,2}$ \\ ${ }^{1}$ Department of Metallurgical Engineering, Kwara State Polytechnic, Ilorin, Nigeria \\ ${ }^{2}$ Department of Materials Science and Engineering, Obafemi Awolowo University, Ile-Ife, Nigeria
}

Email address:

adelekeadedayo58@yahoo.com

To cite this article:

Adeleke Victor Adedayo. Coping with COPE. Science Research. Vol. 2, No. 5, 2014, pp. 98-99. doi: 10.11648/j.sr.20140205.13

\begin{abstract}
This paper identifies and describes a publication attitude which is uprising to some of the COPE best practice guidelines. An overview of COPE was made, one attitude considered as academic misbehaviour by the COPE best practice guidelines was singled out and discussed. Marketing communication strategies were used to establish clear visualizations of the concept of republication to be coping efforts of authors against the confinement of COPE best practice guideline.
\end{abstract}

Keywords: COPE, Coping, Redundant Publication, Duplicate Publication

\section{Introduction}

COPE is the acronym for Committee on Publication Ethics. It is a forum of editors of journal publishing outfits. The purpose of this forum is to discuss issues relevant to strength and honesty of scientific records [1]. Reports, cataloguing and instigation of investigations into ethical problems in the publication process is supported and encouraged.

COPE came into being in 1997 when a group of medical journal editors out of concern, had a forum for promoting good conduct in scientific communications. The first guidelines of COPE were designed by Philip Fulford, Michael Doherty, Jane Smith, Richard Smith, Fiona Godlee, Peter Wilmshurst, Richard Horton and Michael Farting when they had a deliberation at a COPE conference in April of 1999[2]. Also, a COPE code of conduct was drafted in 2004 by Richard Smith who was the then Chair of COPE, and at the same time the Editor of BMJ [2]. The code was assumed to set out standards of good conduct and ethics in scientific communications for guiding against publication misconducts. Some of the identified misconducts include: plagiarism, passing off fraudulent data, unethical research, breaches of confidentiality, publication redundancy. This best practice guideline, however, are more aspirational, and it is recognized that $100 \%$ compliance by stakeholders may not be possible in reality [2]. Stakeholders tend to cope with the confinement set by this best practice guideline. The coping attitudes of individuals involved in the publications process has led to the emergence of other standpoints, such as 'Fair Use', which is contrary to some of the COPE best practice standpoint.

In this paper, redundancy, which is one of the cardinal misconducts identified by COPE, is singled out for more in depth expository study. Some allusions are made to well grasped knowledge in marketing to help in understanding the issues surrounding redundancy as defined by COPE. The aim is to show that labeling duplicate publication as a serious academic misbehavior is a misconstrued idea.

\section{Redundancy and Marketing}

Redundant publication, also popularly referred to as duplicate publication is used to describe a situation where contents of an intellectual material are published more than once by the author. It is considered to be different from plagiarism and copyright violation which involves republication of the contents of an intellectual material by someone who is not the original author. The goal of preventing redundancy is believed to be to engender creativity and novelty. Among a sect of scholars which are referred to as COPE inclined scholars, duplicate publication is considered a very serious academic misbehaviour, however, there is an uprising group that believes that labeling duplicate publication as a serious academic misbehaviour is a misconstrued idea.

Research results can be likened to products from their creator. They can be created for personal needs, also, they can be offered to the public for consumption with or without monetary returns. When products are offered to the public, their effectuality/ returns will be worthwhile when there is effective marketing of the product. The purpose of marketing is to attract attention and create interest. This is done through various means such as advertising, rebranding, targeting, etc. In marketing, every major medium is used to deliver messages 
to promote the product. Different types of media can be attributed to certain kind of groups or life style which makes it possible to appeal to these groups by adapting the contents of the advertisement to capture the identified group. Adapting the content of advertisement to capture identified group is referred to as targeting, which is a very useful approach in establishing authority as the product manufacturer. Advertisement for a particular product can be repackaged severally in order to reestablish the currency of the image of the product. For example, why would Coca-Cola severally repackage the jingles used to advertise Coke? Why not just do a single jingle and let it roll? Why would Coca-Cola use the same advertisement in different media? Why would they make the same advertisement through different television channels and Magazines? Why all these efforts were not considered redundant?

The opinion that the purpose of a research paper is to express claim of furthering knowledge through clear indication of novelty of the research, is misconstrued. Knowledge is furthered through research, however, the goal of publishing a research is to enhance widespread of the knowledge presented in the publication. The primary purpose of publishing is to make information available to the general public for the long term public record. In academic publishing, performance of a published research is often times determined by computing the citation analysis as regards the published research. The visibility of the published research will significantly affect the total citation to the article. Why should efforts geared towards increasing the visibility of a research through some marketing initiative be considered redundancy?

\section{Coping}

In psychology, coping is described as deliberate/conscious effort directed towards solving personal and interpersonal problems. It includes seeking to manage, reduce or tolerate stress or conflict [3-6]. The effectiveness of the coping efforts depends on the type of stress and/or conflict, the particular individual, and the circumstance.

In academic publishing, performance of a publication is often based on number of citations to the publication. The visibility of a scientific communication will significantly affect the total citation to the article. Ordinarily, authors want high performance for their published article. In order to cope with the confining best practice guidelines proposed by COPE, authors of scientific communications resort to deliberate/conscious marketing initiatives directed towards increasing the visibility of their publication. Some of these marketing efforts include re-captioning and repackaging of their published articles for republishing.

\section{Conclusion}

COPE standpoint on redundancy is considered misconstrued. The goal of publishing science research is identified to be to enhance the widespread of the knowledge presented in the publication. In order to increase the visibility of their published works, authors resort to some marketing initiatives which may include re-captioning and repackaging of their publications for republishing.

\section{References}

[1] Elsevier (2014) About COPE, Retrieved from: http://www.elsevier.com/editors/perk/about-cope

[2] COPE (2014) About COPE, Retrieved from: http://publicationethics.org/

[3] Weiten, W., Lloyd, M. A. (2008) Psychology Applied to Modern Life (9th ed.). Wadsworth Canage Learning. ISBN $0-495-55339-5$

[4] Snyder, C. R. (1999) Coping: The Psychology of What Works. New York: Oxford University Press. ISBN 0-19-511934-7

[5] Zeidner, M., Endler, N. S. (1996) Handbook of Coping: Theory, Research, Applications. New York: John Wiley. ISBN 0-471-59946-8

[6] Cummings, E. M., Greene A.L.; Karraker, K. H. (1991) Life Span Developmental Psychology: Perspectives on Stress and Coping, ISBN 978-0-8058-0371-6. 